\title{
Influence of Naloxone and Methysergide on the Analgesic Effects of Low-Level Laser in an Experimental Pain Model
}

\author{
André Peres e Serra ${ }^{1}$, Hazem A Ashmawi ${ }^{2}$
}

\begin{abstract}
Summary: Serra AP, Ashmawi HA - Influence of Naloxone and Methysergide on the Analgesic Effects of Low-Level Laser in an Experimental Pain Model.

Background and objectives: Although the mechanism of action of laser phototherapy (LPT) is not known, it is a promising analgesic method. The aim of this study was to evaluate whether the action of LPT depends on the activation of peripheral opioid or serotonergic receptors.

Method: Inflammatory pain was induced through the injection of carrageenin in the left posterior paw of male Wistar rats. The InGaAIP visible laser

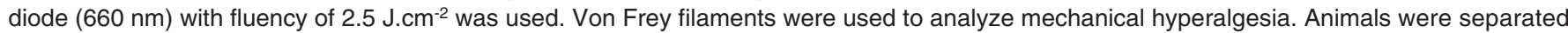
into five groups: Carrageenin; Laser (LPT); Non-coherent light; LPT + Naloxone; and LPT + Methysergide.
\end{abstract}

Results: Low-Level Laser phototherapy proved to be an effective analgesic method, while non-coherent light did not show a similar effect. The use of naloxone blocked the analgesic effect of LPT, while methysergide did not affect LPT-induced analgesia.

Conclusions: According to the parameter used in this study, LPT produced analgesia. Analgesia induced by laser phototherapy is mediated by peripheral opioid receptors. Laser phototherapy does not seem to interact with peripheral serotonergic receptors.

Keywords: ANALGESIA: low-level laser; ANIMALS: Wistar rats.

[Rev Bras Anestesiol 2010;60(3): 302-310] CElsevier Editora Ltda.

\section{INTRODUCTION}

According to the International Association for the Study of Pain (IASP), pain is an unpleasant sensory or emotional experience associated with real or potential tissue damage or described in terms of such damage ${ }^{1}$.

The search for ways to control and treat the different types of pain has been constant. Currently, several methods of analgesia are used. Low-Level Laser (LLL) has been used by surgeons to reduce healing time and comorbidities in the surgical wound, by ophthalmologists to reduce pain caused by glaucoma and treatment of retinopathy of prematurity, by dermatologists in the treatment of lichen planus, dermatitis, and keloids, and by dentists in the treatment of oral mucositis and orofacial pain ${ }^{2-9}$.

Several mechanisms of action have been proposed to explain the therapeutic and analgesic properties of LLL. Analgesia seems to be due to the temporal and spatially coherent light emission of parallel waves of the same length, with synchrony of peaks and valleys ${ }^{10}$. Effects like biomodulation,

Received from the Faculdade de Medicina da Universidade de São Paulo (FMUSP), SP

1. Anesthesiologist; Researcher of the Department of Anesthesiology of the FMUSP

2. Anesthesiologist; Researcher of the Department of Surgery of the FMUSP

Submitted on February 25, 2009

Approved on February 3, 2010

Correspondence to:

Dr. André Peres e Serra

Faculdade de Medicina, Departamento de Cirurgia, Disciplina de Anestesiologia

Av. Dr. Arnaldo, 455

Cerqueira César

01246-903 - São Paulo, SP, Brasil

E-mail:serra88@uol.com.br ability to stimulate cellular division, vasodilation by the release of nitrous oxide, increase in cortisol levels and protein synthesis, and an increase in intracellular calcium and in the activity of superoxide dismutase have also been associated with LLL analgesia 11-16. Another possible mechanism of action would be through the activation of intracellular chromophores present in mitochondria ${ }^{17}$. The hypothesis of three mechanisms of action of LLL has also been suggested: a) photodynamic action on cellular membranes along with an increase in intracellular calcium levels and cellular stimulation; b) photoreactivity of copper-zinc superoxide dismutase (SOD), and c) photolysis of the metallic complexes of nitrous oxide (NO) with the release of this vasodilator, and those characteristics of the laser are responsible for its regenerative and vasodilator effects ${ }^{15}$.

It has also been suggested that singlet oxygen would stimulate RNA and DNA synthesis due to changes in photo absorbing molecules, such as porphyrins and flavoproteins ${ }^{10}$.

The mechanisms mentioned are, mainly, secondary to the intracellular actions of LLL, but it is not clear whether LLL acts directly in the cells or through a second messenger. An anti-inflammatory effect of LLL has been demonstrated ${ }^{18}$. The mediation of the analgesic effect by opioid receptors has been investigated; however, the results are controversial, since opioid receptor-induced ${ }^{18,19}$, as well as opioid receptor independent ${ }^{20}$, effects have been reported. The influence of serotonergic receptors on LLL analgesia has not been reported. One can observe that a consensus on the mechanism of action of LLL does not exist and, therefore, the aim of this study was to investigate the role of opioid and serotonergic receptors on LLL analgesia in an inflammatory pain model induced by carrageenin in rat paw. 


\section{METHODS}

Fifty Wistar male rats weighing 250 to 300 grams, provided by the Vivarium of the Faculdade de Medicina da Universidade de São Paulo (FMUSP) where they were kept for at least 15 days before the study with three animals per compartment for adequate adaptation, being fed with commercial balanced meals and water ad libitum, 12-hour day-light cycle, and temperature ranging from $19^{\circ}$ to $25^{\circ} \mathrm{C}$.

Ethical standards for experiments in conscious animals of the International Association for the Study of Pain (IASP) ${ }^{21}$ were followed. This study was approved by the Research Projects Ethics Committee (CAPPesq) of the Hospital das Clínicas of FMUSP (study registered under number 1513, CAPPesq project number 0956/07). All experiments were undertaken in the Medical Investigation Laboratory 08, FMUSP.

The software Power and Sample Size Program, version 2.1.30 $0^{\circledR}$ was used to calculate the sample size with the following parameters: study power of $80 \%$ to detect differences between means above 0.33 with level of significance of 0.05 , and standard deviation of 0.45 units. Ten rats per group were necessary to fulfill those parameters.

Animals were randomly separated into five groups, with 10 rats per group. Each animal received an injection of 200 $\mu \mathrm{g}$ of carrageenin $\lambda$ (Sigma-Aldrich, Saint Louis, MO, USA) diluted in saline for a total of $100 \mu \mathrm{L}$, in the plantar region of the left hind paw to induce pain ${ }^{22}$. Forty-five minutes after the injection of carrageenin animals were restrained and $50 \mu \mathrm{L}$ of saline was injected in the left hind paw (Control, White Light, and LLL Groups); $1 \mu \mathrm{g} /$ paw of naloxone (Cristália Prod. Quim. Farm., Brazil) in $50 \mu \mathrm{L}$ volume in the posterior hind paw (LLL + Naloxone Group), and $1 \mu \mathrm{g} /$ paw of methysergide (SigmaAldrich, Saint Louis, MO, USA) in $50 \mu \mathrm{L}$ volume in the left hind paw (LLL + Methysergide Group).
Fifteen minutes after the administration of saline, naloxone, or methysergide, according to the group, the animals received the first application of white light or LLL. The second application was done one hour after the first one, according to the information in Box I.

A semiconductor InGaAIP visible laser diode (Photon Lase III, DMC, São Carlos, Brazil) with wavelength of $660 \mathrm{~nm}$, fluency of $2.5 \mathrm{J.cm}^{-2}$ was used based on the following parameters: potency of $15 \times 10^{-3} \mathrm{~W}$, tip with $0.028 \mathrm{~cm}^{2}, 2 \mathrm{~mm}$ from the skin of the rat paw for 5 seconds ${ }^{14,23}$.

Von Frey filaments were used to evaluate carrageenininduced mechanical hyperalgesia. Animals were placed over a raised plastic mesh $(21 \times 27 \times 15 \mathrm{~cm})$ with $12 \times 12 \mathrm{~mm}$ openings, with a transparent plastic cover and, after the adaptation period, they were tested with Frey nylon filaments (Stoelting Co, Wood Dale, IL, USA). Filaments were applied, perpendicular to the paw of the animal, through the openings of the plastic mesh, with increasing force, between $10 \mathrm{mN}$ and $250 \mathrm{mN}$. If an animal did not show any response to the $250 \mathrm{mN}$ filament, the use of the $522 \mathrm{mN}$ (the following filament) filament was considered. Three measurements, with intervals of 5 and 10 minutes, were obtained and the smallest of the three measurements was considered the threshold. Measurements were performed in both hind paws before the administration of the antagonists and 1 hour, 2 hours, 3 hours, and 4 hours after the administration of carrageenin.

The generalized linear model (GLM), a type of statistical test used to compare the curves obtained as a whole, was used for the statistical analysis. Analysis of Variance (ANOVA) was used to compare the results at each moment $(0$, $1,2,3$, and 4 hours). The level of significance smaller than five per cent $(p<0.05)$ was adopted. The statistical analysis software SPSS 13.0 was used. Results were expressed as mean \pm SEM

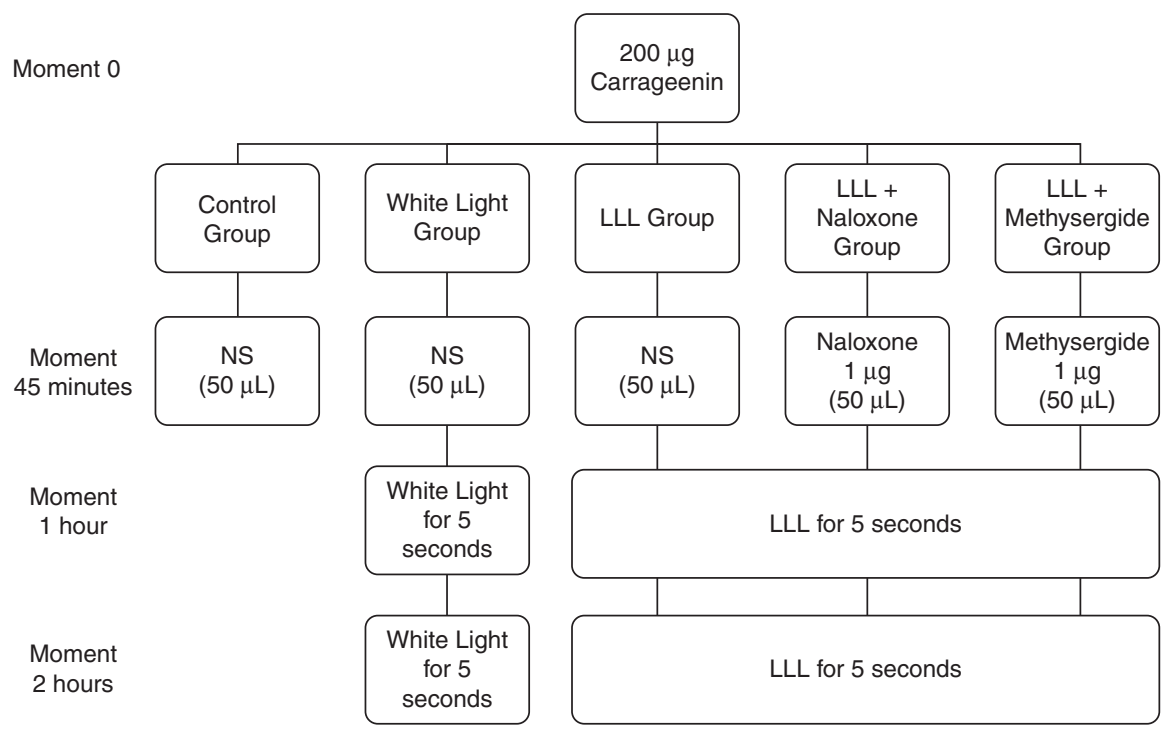

Box I - LLL: Low-Level Laser 
Carrageenin, saline, and antagonists were administered by the same investigator (APS), who also performed the algesimetric test.

\section{RESULTS}

Table I shows the results obtained by the generalized linear model (GLM). The results presented are related with the effects of group interaction and time, i.e., comparing the curves as a whole always between two groups.

Laser Group - it was different from the Control group in the generalized linear method analysis $(p=0.032)$. Comparing the results between different hours, a significant difference was observed at 3 and 4 hours when compared to the Control group (Chart 1).

White Light Group - it did not differ from the control group in the generalized linear model analysis, but it differed from the Laser group. Using the hour-to-hour comparison, the difference between both groups was seen at the fourth hour after the administration of carrageenin (Chart I).

Laser + Naloxone Group - the LLL + Naloxone group did not differ from the control group, but a significant difference was observed when it was compared to the Laser group, with an increase in hyperalgesia. Comparing the hour-to-hour results, a difference was observed at moments 3 and 4 hours in relation to the Laser group (Chart 1).

Laser + Methysergide Group - the LLL + Methysergide group differed from the Control group in the generalized line-

Table I - Descriptive analysis Obtained by the Generalized Linear Method (GLM)

\begin{tabular}{llll}
\hline Group 1 & Group 2 & DL & p \\
\hline Control & LLL & 4 & $0.032^{*}$ \\
Control & White light & 4 & 0.385 \\
LLL + Methysergide & Control & 4 & $0.001^{*}$ \\
LLL + Methysergide & LLL & 4 & 0.563 \\
LLL + Naloxone & Control & 4 & 0.979 \\
LLL + Naloxone & LLL & 4 & $0.014^{*}$
\end{tabular}

$\mathrm{DL}-$ degrees of liberty ${ }^{*} p<0.05$. ar model analysis, but differences were not observed when it was compared to the Laser group. Comparing the results hour-to-hour, the difference was observed at 2, 3, and 4 hours (Chart 1).

\section{DISCUSSION}

In the search for new analgesic and anti-inflammatory methods Low-Level Laser (LLL) seems promising, since it is effective in reducing inflammatory pain with few side effects ${ }^{18}$. The effects of LLL are dose-dependent, and a response is not observed when sub- or super-dosage is used, but effective analgesia is observed when used in adequate doses ${ }^{14,23}$. As expected, the LLL in the parameters used here proved to produce analgesia, increasing significantly the mechanical hyperalgia threshold in animals in the Laser Group when compared to the Control Group. The White Light Group (source of common non-monochromatic, non-coherent light) used as a second control did not produce analgesia either.

The interaction between LLL-induced analgesia and opioid receptors is controversial. Low-Level Laser increases the peripheral release of opioids through migration of immune system cells, with local release of beta-endorphin, which is antagonized by naloxone ${ }^{18,19}$; on the other hand, another study using a carrageenin-induced pain model did not demonstrate that the effects of LLL were antagonized by naloxone ${ }^{20}$. The models and laser doses used differed among the studies, as well as the route of naloxone administration, intraperitoneal or intraplantar ${ }^{20}$. The present study demonstrated mediation of the analgesia by naloxone, which is similar to the results of previous studies ${ }^{18,19}$.

Naloxone has an onset of action of two minutes, with duration of action dependent on the dose and route of administration. It has a half-life ranging from 43 to 90 minutes, and duration of action of approximately 1.5 hour 24,25 . In the present study, naloxone was applied 15 minutes before the first dose of LLL and 1h15min before the second dose, and its concentrations were adequate during the applications of the LLL. Although naloxone crosses the blood-brain barrier, in the dose of $1 \mu \mathrm{g} / \mathrm{paw}$ it only antagonizes peripheral opioid effects without a systemic effect 26,27 .

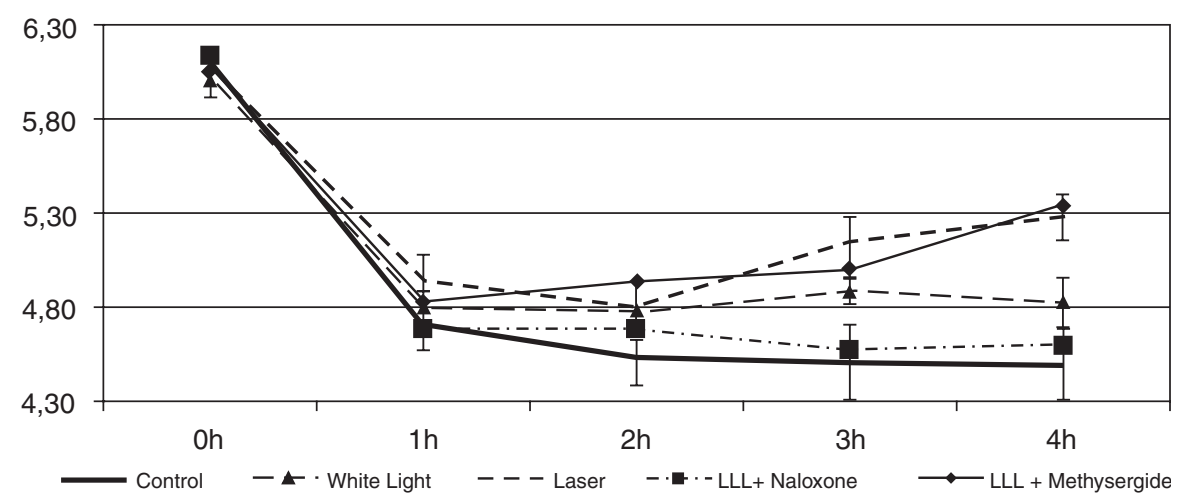

Chart 1 - Paw Withdrawal Threshold with von Frey Filaments (Log grams-force). 
Naloxone is a non-specific opioid receptor antagonist, with higher affinity for $\mu$ and $<$ receptors and lower affinity for $\delta$ receptors. As a function of the higher affinity for $\mu$ and $<$ receptors, it is possible that the analgesic effect of LLL is mediated by those two receptors. Since the affinity of naloxone for $\delta$ receptors is smaller, it is not possible to determine the role of this receptor in LLL-induced analgesia, and the use of a specific antagonist would be interesting.

The role of serotonin (5-HT) on LLL analgesia had not been investigated. Serotonin has several different types of receptors $\left(5-\mathrm{HT}_{1}, 5-\mathrm{HT}_{2}, 5-\mathrm{HT}_{3}, 5-\mathrm{HT}_{4}, 5-\mathrm{HT}_{5}, 5-\mathrm{HT}_{6}, 5-\mathrm{HT}_{7}\right)$ in different tissues with different functions. In the peripheral nervous system, serotonin has been associated with the pronociceptive effect 28,29 . However, it has been indicated that the $5-\mathrm{HT}_{1}$ receptor is pro-nociceptive and analgesic ${ }^{29,30}$. Due to this uncertainty, we used methysergide, an antagonist of $5-\mathrm{HT}_{1}, 5-\mathrm{HT}_{2}$, and $5-\mathrm{HT}_{7}$ receptors, to determine whether LLLinduced analgesia is mediated by peripheral $5-\mathrm{HT}_{1}$ receptors, which was not confirmed by the results observed, in which the analgesic pattern of LLL is maintained in the presence of me- thysergide. Since the effects of the LLL were not potentiated either, the evidence indicates that its analgesic function is independent of peripheral $5-\mathrm{HT}_{1}, 5-\mathrm{HT}_{2}$, and $5-\mathrm{HT}_{7}$ receptors.

The results of the present study support, at least partially, the hypothesis that LLL-mediated analgesia is mediated by peripheral opioid receptors, but not by peripheral serotonergic receptors.

\section{ACKNOWLEDGEMENTS}

We would like to acknowledge Professor Ana Cláudia Luiz and Professor Alyne Simões, MD, of the Special Odontology Laser Laboratory of the Odontology school of USP (LELOFOUSP) for their help on using and obtaining the laser equipment. To Mr. Gilberto, a technician of the Medical Investigation Laboratory 08 of the Faculdade de Medicina da USP, for his help with the experiments. And to DMC Equipamentos Ltda. (São Carlos, SP, Brazil) for the lending of the equipment Photon Lase III used in the present study. 